Bull. Korean Math. Soc. 52 (2015), No. 6, pp. 1925-1935

http://dx.doi.org/10.4134/BKMS.2015.52.6.1925

\title{
A NOTE ON CONVEXITY OF CONVOLUTIONS OF HARMONIC MAPPINGS
}

\author{
Yue-Ping Jiang, Antti Rasila, and Yong Sun
}

\begin{abstract}
In this paper, we study right half-plane harmonic mappings $f_{0}$ and $f$, where $f_{0}$ is fixed and $f$ is such that its dilatation of a conformal automorphism of the unit disk. We obtain a sufficient condition for the convolution of such mappings to be convex in the direction of the real axis. The result of the paper is a generalization of the result of by $\mathrm{Li}$ and Ponnusamy [11], which itself originates from a problem posed by Dorff et al. in [7].
\end{abstract}

\section{Introduction}

Let $\mathbb{D}=\{z \in \mathbb{C}:|z|<1\}$ be the unit disk. We will consider the family of complex-valued harmonic mapping $f=u+i v$ defined in a domain $D \subset \mathbb{C}$ if $u$ and $v$ are real harmonic in $D$, i.e., $\Delta u=\Delta v=0$, where $\Delta$ is the complex Laplacian operator

$$
\Delta=4 \frac{\partial^{2}}{\partial z \partial \bar{z}}:=\frac{\partial^{2}}{\partial x^{2}}+\frac{\partial^{2}}{\partial y^{2}} .
$$

Denote by $\mathcal{H}$ the class of all complex-valued harmonic mappings $f$ in $\mathbb{D}$ normalized by $f(0)=f_{z}(0)-1=0$. Let $\mathcal{S}_{H}$ be the subclass of $\mathcal{H}$ consisting of univalent and sense-preserving functions. For a simply connected domain $\mathbb{D}$, such functions can be written in the form $f=h+\bar{g}$, where

$$
h(z)=z+\sum_{n=2}^{\infty} a_{n} z^{n} \quad \text { and } \quad g(z)=\sum_{n=1}^{\infty} b_{n} z^{n}
$$

are analytic (cf. [8]) in $\mathbb{D}$ and the Jacobian $J_{f}(z)=\left|h^{\prime}(z)\right|^{2}-\left|g^{\prime}(z)\right|^{2}>0$, or equivalently there exists an analytic complex dilatation $\omega$ of $f$ such that

$$
|\omega(z)|=\left|\frac{g^{\prime}(z)}{h^{\prime}(z)}\right|<1 \quad\left(h^{\prime}(z) \neq 0 ; z \in \mathbb{D}\right) .
$$

The classical family $\mathcal{S}$ of analytic univalent and normalized functions in $\mathbb{D}$ is a subclass of $\mathcal{S}_{H}$ with $g(z) \equiv 0$. The family of all functions $f \in \mathcal{S}_{H}$ with the

Received August 19, 2014; Revised June 11, 2015.

2010 Mathematics Subject Classification. Primary 30C45; Secondary 30C20, 30C65.

Key words and phrases. harmonic univalent mapping, convolution, half-plane mapping, convex function. 
additional property that $f_{\bar{z}}(0)=0$ is denoted by $\mathcal{S}_{H}^{0}$. We let $\mathcal{K}_{H}^{0}, \mathcal{S}_{H}^{* 0}$ and $\mathcal{C}_{H}^{0}$ denote the subclasses of $\mathcal{S}_{H}^{0}$ mapping $\mathbb{D}$ onto convex, starlike and close-toconvex domains, respectively.

A domain $\Omega \subset \mathbb{C}$ is said to be convex in the direction $\gamma$ if for all $a \in \mathbb{C}$, the set $\Omega \cap\left\{a+t e^{i \gamma}: t \in \mathbb{R}\right\}$ is either connected or empty. In particular, a domain is convex in the direction of the real (or imaginary) axis if every line parallel to the real (or imaginary) axis has a connected intersection with the domain.

For two harmonic functions

$$
f(z)=h(z)+\overline{g(z)}=z+\sum_{n=2}^{\infty} a_{n} z^{n}+\sum_{n=1}^{\infty} \overline{b_{n}} \bar{z}^{n}
$$

and

$$
F(z)=H(z)+\overline{G(z)}=z+\sum_{n=2}^{\infty} A_{n} z^{n}+\sum_{n=1}^{\infty} \overline{B_{n}} \bar{z}^{n},
$$

their harmonic convolution is denoted by $f * F$ and defined as follows:

$$
f * F=h * H+\overline{g * G}=z+\sum_{n=2}^{\infty} a_{n} A_{n} z^{n}+\sum_{n=1}^{\infty} \overline{b_{n} B_{n}} \bar{z}^{n} .
$$

For recent investigations on harmonic convolution, see e.g. [1, 2, 4, 6, 7, 8, 9, $10,11,12]$, and references therein.

A function $f=h+\bar{g} \in \mathcal{S}_{H}^{0}$ is called a right half-plane mapping if $f$ maps $\mathbb{D}$ onto $\{w: \Re(w)>-1 / 2\}$. Such a mapping can be presented in the form

$$
h(z)+g(z)=\frac{z}{1-z} \quad(z \in \mathbb{D}) .
$$

We denote by $\mathcal{R}_{H}^{0}$, the class of all half-plane mappings, and note that $\mathcal{R}_{H}^{0} \subseteq$ $\mathcal{K}_{H}^{0}$. Specifically, for $f_{0}=h_{0}+\overline{g_{0}} \in \mathcal{R}_{H}^{0}$ with the dilatation $\omega_{0}=-z$, by applying the shearing technique, we obtain the canonical right half-plane mapping with

$$
h_{0}=\frac{z-\frac{1}{2} z^{2}}{(1-z)^{2}}=\frac{1}{2}\left(\frac{z}{1-z}+\frac{z}{(1-z)^{2}}\right)
$$

and

$$
g_{0}=\frac{-\frac{1}{2} z^{2}}{(1-z)^{2}}=\frac{1}{2}\left(\frac{z}{1-z}-\frac{z}{(1-z)^{2}}\right) .
$$

For the convolution of analytic functions, if $f_{1}, f_{2} \in K$, then $f_{1} * f_{2} \in K$. Also, the right half-plane mapping, $z /(1-z)$, acts as the convolution identity. In the harmonic case, there are infinitely many right half-plane mappings and the harmonic convolution of one of these right half-plane mappings with a function $f \in \mathcal{K}_{H}^{0}$ may not preserve the properties of $f$, such as convexity or even univalence (cf. [6]).

However, the results below guarantee that the harmonic convolution of a right half-plane mapping with another harmonic mapping with special dilatation will at least be convex in the direction of the real axis. 
Theorem $\mathbf{A}([6])$. Let $f_{1}=h_{1}+\overline{g_{1}}, f_{2}=h_{2}+\overline{g_{2}} \in \mathcal{R}_{H}^{0}$. If $f_{1} * f_{2}$ is locally univalent and sense-preserving, then $f_{1} * f_{2} \in \mathcal{S}_{H}^{0}$ and is convex in the direction of the real axis.

Theorem B ([7]). Let $f=h+\bar{g} \in \mathcal{R}_{H}^{0}$ with the dilatation $\omega(z)=e^{i \theta} z^{n}$, where $n \in \mathbb{Z}^{+}$and $\theta \in \mathbb{R}$. If $n=1,2$, then $f_{0} * f \in \mathcal{S}_{H}^{0}$ and is convex in the direction of the real axis.

Theorem C ([7]). Let $f=h+\bar{g} \in \mathcal{R}_{H}^{0}$ with the dilatation $\omega(z)=\frac{z+a}{1+a z}$, where $a \in(-1,1)$. Then $f_{0} * f \in \mathcal{S}_{H}^{0}$ and is convex in the direction of the real axis.

Recently, Bshouty and Lyzzaik [3] presented a collection of problems and conjectures in planar harmonic mappings. The problem 3.26(a) of Dorff et al. [7] is given below.

Problem. Let $f=h+\bar{g} \in \mathcal{R}_{H}^{0}$ with the dilatation $\omega(z)=\frac{z+a}{1+\bar{a} z},|a|<1$. Determine other values of $a \in \mathbb{D}$ for which the result of Theorem $\mathrm{C}$ holds.

Recently, Li and Ponnusamy [11] have solved this problem. Their result is the following.

Theorem D. Let $f=h+\bar{g} \in \mathcal{R}_{H}^{0}$ with the dilatation $\omega(z)=\frac{z+a}{1+\bar{a} z},|a|<1$. Then $f_{0} * f \in \mathcal{S}_{H}^{0}$ and is convex in the direction of the real axis if and only if

$$
(\Re(a))^{2}+9(\Im(a))^{2} \leq 1 \quad \text { and } \quad \Re(a) \neq \pm 1 .
$$

Furthermore, Li and Ponnusamy [12] have considered this result in a more general setting by allowing $f$ to be a slanted half-plane harmonic mapping.

In this paper, we consider the above problem in another more general setting, where $\omega$ is allowed to be the conformal mapping of the unit disk onto itself of the form

$$
\omega(z)=e^{i \theta} \frac{z+a}{1+\bar{a} z} \quad(\theta \in \mathbb{R} ;|a|<1) .
$$

Our main result is Theorem 1, which gives a sufficient condition for the mapping $f_{0} * f \in \mathcal{S}_{H}^{0}$ and to be convex in the direction of the real axis with this more general dilatation, thus improving Theorem B, Theorem $\mathrm{C}$, and the sufficiency part of Theorem D. Finally, we give an example illustrating potential applications of our main result, and showing that our result does not follow from Theorem D.

\section{Preliminary results}

The proofs of our main results are based on the following lemmas.

Lemma 1 ([6]). Let $f=h+\bar{g} \in \mathcal{R}_{H}^{0}$ with the dilatation $\omega(z)$. Then the dilatation $\widetilde{\omega}$ of $f_{0} * f$ is

$$
\widetilde{\omega}(z)=-z \frac{\omega^{2}(z)+\omega(z)-\frac{1}{2} z \omega^{\prime}(z)+\frac{1}{2} \omega^{\prime}(z)}{1+\omega(z)-\frac{1}{2} z \omega^{\prime}(z)+\frac{1}{2} z^{2} \omega^{\prime}(z)} .
$$


Lemma 2. Let $f=h+\bar{g} \in \mathcal{R}_{H}^{0}$ with the dilatation $\omega(z)$ be defined by (6). Then the dilatation $\widetilde{\omega}$ of $f_{0} * f$ is

$$
\widetilde{\omega}(z)=-z e^{i \phi} \frac{t(z)}{t^{*}(z)}=-z e^{i \phi} \frac{(z+A)(z+B)}{(1+\bar{A} z)(1+\bar{B} z)},
$$

where $\phi=\arg \left(\left(\bar{a}+e^{i \theta}\right) /\left(a+e^{-i \theta}\right)\right)$,

$$
t(z)=z^{2}+\frac{4 a e^{i \theta}+3|a|^{2}+1}{2\left(\bar{a}+e^{i \theta}\right)} z+\frac{2 a^{2} e^{i \theta}+2 a+1-|a|^{2}}{2\left(\bar{a}+e^{i \theta}\right)}
$$

and

(10) $t^{*}(z)=z^{2} \overline{t(1 / \bar{z})}=1+\frac{4 \bar{a} e^{-i \theta}+3|a|^{2}+1}{2\left(a+e^{-i \theta}\right)} z+\frac{2 \bar{a}^{2} e^{-i \theta}+2 \bar{a}+1-|a|^{2}}{2\left(a+e^{-i \theta}\right)} z^{2}$.

Here $-A,-B$ are the two roots of the equation $t(z)=0$, and $A, B$ may be equal.

Proof. In view of (6), we have

$$
\omega^{\prime}(z)=e^{i \theta} \frac{1-|a|^{2}}{(1+\bar{a} z)^{2}} .
$$

By Lemma 1 and (6), the expression for $\widetilde{\omega}(z)$ given by $(7)$ takes the form

$$
\widetilde{\omega}(z)=-z \frac{\omega^{2}(z)+\omega(z)-\frac{1}{2} z \omega^{\prime}(z)+\frac{1}{2} \omega^{\prime}(z)}{1+\omega(z)-\frac{1}{2} z \omega^{\prime}(z)+\frac{1}{2} z^{2} \omega^{\prime}(z)}=-z\left(\frac{\bar{a}+e^{i \theta}}{a+e^{-i \theta}}\right) \frac{t(z)}{t^{*}(z)} .
$$

Here $t(z)$ and $t^{*}(z)$ are given by (9) and (10), respectively. Suppose that $-A,-B$ are the two roots of $t(z)=0$. Then

$$
t(z)=(z+A)(z+B)
$$

and

$$
t^{*}(z)=z^{2} \overline{t(1 / \bar{z})}=z^{2} \overline{(1 / \bar{z}+A)(1 / \bar{z}+B)}=(1+\bar{A} z)(1+\bar{B} z) .
$$

As $\left|\left(\bar{a}+e^{i \theta}\right) /\left(a+e^{-i \theta}\right)\right|=1$, the desired form for $\widetilde{\omega}(z)$ follows.

Lemma 3. Let $t(z)$ be defined by (9) and write $t(z)=(z+A)(z+B)$. Also, let $a=|a| e^{i \alpha}$, where $\alpha=\arg a$ with $|a|<1$. If

$$
\left[9 \sin ^{2}\left(\alpha+\frac{\theta}{2}\right)+\cos ^{2}\left(\alpha+\frac{\theta}{2}\right)\right]|a|^{2} \leq 1,
$$

then $|A B| \leq 1$. Moreover, $|A B|=1$ if and only if

(12) $|a| \cos \left(\alpha+\frac{\theta}{2}\right)=-\cos \frac{\theta}{2}$ and $3|a| \sin \left(\alpha+\frac{\theta}{2}\right)=\sin \frac{\theta}{2}$ for $\frac{1}{3} \leq|a|<1$. 
Proof. By the definition of $t(z)$, it is clear that

$$
A B=\frac{2 a^{2} e^{i \theta}+2 a+1-|a|^{2}}{2\left(\bar{a}+e^{i \theta}\right)}=\frac{2 a\left(a e^{i \theta}+1\right)+\left(1-|a|^{2}\right)}{2\left(\bar{a}+e^{i \theta}\right)} .
$$

A computation leads to

$$
\left|2 a\left(a e^{i \theta}+1\right)+\left(1-|a|^{2}\right)\right|^{2}-4\left|\bar{a}+e^{i \theta}\right|^{2}=\left(1-|a|^{2}\right) v(a),
$$

where $v(a)$ is real and

$$
v(a)=2\left(a^{2} e^{i \theta}+\bar{a}^{2} e^{-i \theta}\right)+2(a+\bar{a})-4\left(a e^{i \theta}+\bar{a} e^{-i \theta}\right)-3-5|a|^{2} .
$$

Suppose that $a=|a| e^{i \alpha}$, and by a simplification, we have

$$
\begin{aligned}
v(a)= & 4|a|^{2} \cos (2 \alpha+\theta)+4|a| \cos \alpha-8|a| \cos (\alpha+\theta)-3-5|a|^{2} \\
= & 4|a|^{2}\left[1-2 \sin ^{2}\left(\alpha+\frac{\theta}{2}\right)\right] \\
& +4|a|\left[\cos \left(\alpha+\frac{\theta}{2}\right) \cos \frac{\theta}{2}+\sin \left(\alpha+\frac{\theta}{2}\right) \sin \frac{\theta}{2}\right] \\
& -8|a|\left[\cos \left(\alpha+\frac{\theta}{2}\right) \cos \frac{\theta}{2}-\sin \left(\alpha+\frac{\theta}{2}\right) \sin \frac{\theta}{2}\right]-3-5|a|^{2} \\
= & 1-\left[|a| \cos \left(\alpha+\frac{\theta}{2}\right)+2 \cos \frac{\theta}{2}\right]^{2}-\left[3|a| \sin \left(\alpha+\frac{\theta}{2}\right)-2 \sin \frac{\theta}{2}\right]^{2} .
\end{aligned}
$$

By virtue of (11), we observe that

$$
P_{1}(x, y)=P_{1}\left(|a| \cos \left(\alpha+\frac{\theta}{2}\right), 3|a| \sin \left(\alpha+\frac{\theta}{2}\right)\right)
$$

is a point that lies in the closed unit disk

$$
\left\{z=x+y i \in \mathbb{C}: x^{2}+y^{2} \leq 1\right\}
$$

whereas the point $P_{2}\left(-2 \cos \frac{\theta}{2}, 2 \sin \frac{\theta}{2}\right)$ lies on the circle $|z|=2$. Thus, the distance between the points $P_{1}$ and $P_{2}$ must be at least 1 . That is,

$$
\sqrt{\left[|a| \cos \left(\alpha+\frac{\theta}{2}\right)+2 \cos \frac{\theta}{2}\right]^{2}+\left[3|a| \sin \left(\alpha+\frac{\theta}{2}\right)-2 \sin \frac{\theta}{2}\right]^{2}} \geq 1
$$

which is equivalent to saying that $v(a) \leq 0$, i.e., $|A B| \leq 1$. Moreover, in the above inequality, equality holds if and only if the point $P_{1}$ is the middle point of the line segment joining $P_{2}$ and the origin, that is $P_{1}(x, y)$ satisfies $x^{2}+y^{2}=1$. Since we note that $P_{1}(x, y)$ also is a point lies on the ellipse

$$
\left\{z=x+y i \in \mathbb{C}: \frac{x^{2}}{|a|^{2}}+\frac{y^{2}}{9|a|^{2}}=1 \text { for } 0<|a|<1\right\} .
$$


Thus, equality holds if and only if the point $P_{1}(x, y)$ satisfies the following system of equations

$$
\left\{\begin{aligned}
x^{2}+y^{2} & =1 \\
\frac{x^{2}}{|a|^{2}}+\frac{y^{2}}{9|a|^{2}} & =1
\end{aligned}\right.
$$

and for $1 / 3 \leq|a|<1$, the points

$$
P_{1}\left(|a| \cos \left(\alpha+\frac{\theta}{2}\right), 3|a| \sin \left(\alpha+\frac{\theta}{2}\right)\right)=P_{1}\left( \pm \sqrt{\frac{9|a|^{2}-1}{8}}, \pm \sqrt{\frac{9-9|a|^{2}}{8}}\right)
$$

are the real roots of the above system of equations. That is that $|A B|=1$ if and only if (12) holds. In conclusion, if (11) holds but not the (12), then $v(a)<0$ and hence, $|A B|<1$ holds. If (12) holds, then $v(a)=0$ and hence, $|A B|=1$ holds. This completes the proof.

Lemma 4 (Cohn's Rule, see [5]). Given a polynomial

$$
f(z)=a_{0}+a_{1} z+\cdots+a_{n} z^{n}
$$

of degree $n$, let

$$
f^{*}(z)=z^{n} \overline{f(1 / \bar{z})}=\overline{a_{n}}+\overline{a_{n-1}} z+\cdots+\overline{a_{0}} z^{n} .
$$

Denote by $p$ and $s$ the number of zeros of $f$ inside the unit circle and on it, respectively. If $\left|a_{0}\right|<\left|a_{n}\right|$, then

$$
f_{1}(z)=\frac{\overline{a_{n}} f(z)-a_{0} f^{*}(z)}{z}
$$

is of degree $n-1$ with $p_{1}=p-1$ and $s_{1}=s$ the number of zeros of $f_{1}$ inside the unit circle and on it, respectively.

\section{Main results}

The main result of this paper is a generalization of the result of $\mathrm{Li}$ and Ponnusamy. The difference is that our result allows a rotation parameter $\theta$ in the dilatation.

Theorem 1. Let $f=h+\bar{g} \in \mathcal{R}_{H}^{0}$ with the dilatation $\omega(z)=e^{i \theta} \frac{z+a}{1+\bar{a} z}$, where $a=|a| e^{i \alpha}, \alpha=\arg a,|a|<1$ and $\theta \in \mathbb{R}$. If

$$
\left[9 \sin ^{2}\left(\alpha+\frac{\theta}{2}\right)+\cos ^{2}\left(\alpha+\frac{\theta}{2}\right)\right]|a|^{2} \leq 1
$$

and

$$
|a| \cos \left(\alpha+\frac{\theta}{2}\right) \neq-\cos \frac{\theta}{2}
$$

then $f_{0} * f \in \mathcal{S}_{H}^{0}$ and is convex in the direction of the real axis. 
Proof. By Lemma 2 and the hypothesis, we can get the dilatation $\widetilde{\omega}(z)$ of $f_{0} * f$ has the form (8). Then, we consider the function $t(z)$ defined by (9) in the form

$$
t(z)=z^{2}+a_{1} z+a_{0}=(z+A)(z+B),
$$

where $-A,-B$ are the two roots of the equation $t(z)=0$, so that

$$
A+B=a_{1}=\frac{4 a e^{i \theta}+3|a|^{2}+1}{2\left(\bar{a}+e^{i \theta}\right)} \quad \text { and } \quad A B=a_{0}=\frac{2 a^{2} e^{i \theta}+2 a+1-|a|^{2}}{2\left(\bar{a}+e^{i \theta}\right)} \text {. }
$$

By virtue of Lemma 3 and the hypothesis, we have $\left|a_{0}\right|=|A B|<1$. Thus, for a given $a \in \mathbb{D}$, at least one of $-A,-B$ lies in $\mathbb{D}$. Now, without loss of generality, we may assume that $-A \in \mathbb{D}$. Furthermore, we can use Cohn's rule establish the result.

We note that

$$
t_{1}(z)=\frac{t(z)-a_{0} t^{*}(z)}{z}=\left(1-\left|a_{0}\right|^{2}\right) z+a_{1}-a_{0} \overline{a_{1}}
$$

and so, $t_{1}(z)$ has one zero at

$$
z_{0}=\frac{a_{0} \overline{a_{1}}-a_{1}}{1-\left|a_{0}\right|^{2}}=\frac{A\left(|B|^{2}-1\right)+B\left(|A|^{2}-1\right)}{1-|A B|^{2}} .
$$

Now, we will prove the claim that $-B \in \overline{\mathbb{D}}$ if and only if $\left|z_{0}\right| \leq 1$. As the assumption $|A|<1$, and the relation $|A B|<1$, and by a routine computation, it can be easily seen that

$\left|A\left(|B|^{2}-1\right)+B\left(|A|^{2}-1\right)\right|^{2}-\left(1-|A B|^{2}\right)^{2}=-\left(1-|A|^{2}\right)\left(1-|B|^{2}\right)|1-A \bar{B}|^{2} \leq 0$ if and only if $|B| \leq 1$. In view of (16), it shows that $|B|<1$ if and only if $\left|z_{0}\right|<1$, and $|B|=1$ if and only if $\left|z_{0}\right|=1$.

Next, we need to simplify the expression for $z_{0}$. After a cumbersome calculation and simplification, we may conveniently write $z_{0}$ as

$$
z_{0}=\frac{a_{0} \overline{a_{1}}-a_{1}}{1-\left|a_{0}\right|^{2}}=\frac{u(a)}{v(a)}
$$

where $v(a)$ is given by (13) and

$$
u(a)=6 a^{2} e^{i \theta}-4 \bar{a} e^{-i \theta}+8 a+2 e^{-i \theta}-1-3|a|^{2} .
$$

We observe that $\left|z_{0}\right| \leq 1$ if and only if $|u(a)|^{2}-|v(a)|^{2} \leq 0$. In order to deal with the inequality, as $v(a)$ is real, we will consider

$$
|u(a)|^{2}-|v(a)|^{2}=[\Re(u(a))-v(a)][\Re(u(a))+v(a)]+[\Im(u(a))]^{2},
$$

and let

First we find that

$$
a=|a| e^{i \alpha} \quad(|a|<1 ; \alpha \in \mathbb{R}) .
$$

$\Re(u(a))=6|a|^{2} \cos (2 \alpha+\theta)-4|a| \cos (\alpha+\theta)+8|a| \cos \alpha+2 \cos \theta-1-3|a|^{2}$, 
and

$$
\begin{aligned}
\Im(u(a))= & 6|a|^{2} \sin (2 \alpha+\theta)+4|a| \sin (\alpha+\theta)+8|a| \sin \alpha-2 \sin \theta \\
= & 12|a|^{2} \sin \left(\alpha+\frac{\theta}{2}\right) \cos \left(\alpha+\frac{\theta}{2}\right)+12|a| \sin \left(\alpha+\frac{\theta}{2}\right) \cos \frac{\theta}{2} \\
& -4|a| \cos \left(\alpha+\frac{\theta}{2}\right) \sin \frac{\theta}{2}-4 \sin \frac{\theta}{2} \cos \frac{\theta}{2} \\
= & 4\left[|a| \cos \left(\alpha+\frac{\theta}{2}\right)+\cos \frac{\theta}{2}\right]\left[3|a| \sin \left(\alpha+\frac{\theta}{2}\right)-\sin \frac{\theta}{2}\right] .
\end{aligned}
$$

Also, we see that

$$
\begin{aligned}
& \Re(u(a))-v(a) \\
= & 2\left[|a|^{2} \cos (2 \alpha+\theta)+2|a| \cos (\alpha+\theta)+2|a| \cos \alpha+\cos \theta+|a|^{2}+1\right] \\
= & 2\left[|a|^{2}(\cos (2 \alpha+\theta)+1)+2|a|(\cos (\alpha+\theta)+\cos \alpha)+(\cos \theta+1)\right] \\
= & 4\left[|a|^{2} \cos ^{2}\left(\alpha+\frac{\theta}{2}\right)+2|a| \cos \left(\alpha+\frac{\theta}{2}\right) \cos \frac{\theta}{2}+\cos ^{2} \frac{\theta}{2}\right] \\
= & 4\left[|a| \cos \left(\alpha+\frac{\theta}{2}\right)+\cos \frac{\theta}{2}\right]^{2}
\end{aligned}
$$

and similarly,

$$
\begin{aligned}
& \Re(u(a))+v(a) \\
= & 10|a|^{2} \cos (2 \alpha+\theta)-12|a| \cos (\alpha+\theta)+12|a| \cos \alpha+\cos \theta-8|a|^{2}-4 \\
= & 2|a|^{2}[5 \cos (2 \alpha+\theta)-4]+12|a|[\cos \alpha-\cos (\alpha+\theta)]+2(\cos \theta-2) \\
= & 2|a|^{2}\left[1-10 \sin ^{2}\left(\alpha+\frac{\theta}{2}\right)\right]+24|a| \sin \left(\alpha+\frac{\theta}{2}\right) \sin \frac{\theta}{2}-4 \sin ^{2} \frac{\theta}{2}-2 .
\end{aligned}
$$

Using the above formulas, we get

$$
\begin{aligned}
& |u(a)|^{2}-|v(a)|^{2} \\
= & 8\left[|a| \cos \left(\alpha+\frac{\theta}{2}\right)+\cos \frac{\theta}{2}\right]^{2}\left\{2\left(3|a| \sin \left(\alpha+\frac{\theta}{2}\right)-\sin \frac{\theta}{2}\right)^{2}\right. \\
& \left.+|a|^{2}\left[1-10 \sin ^{2}\left(\alpha+\frac{\theta}{2}\right)\right]+12|a| \sin \left(\alpha+\frac{\theta}{2}\right) \sin \frac{\theta}{2}-2 \sin ^{2} \frac{\theta}{2}-1\right\} \\
= & 8\left[|a| \cos \left(\alpha+\frac{\theta}{2}\right)+\cos \frac{\theta}{2}\right]^{2}\left[|a|^{2}+8|a|^{2} \sin ^{2}\left(\alpha+\frac{\theta}{2}\right)-1\right] \\
= & 8\left[|a| \cos \left(\alpha+\frac{\theta}{2}\right)+\cos \frac{\theta}{2}\right]^{2}\left\{\left[9 \sin ^{2}\left(\alpha+\frac{\theta}{2}\right)+\cos ^{2}\left(\alpha+\frac{\theta}{2}\right)\right]|a|^{2}-1\right\} .
\end{aligned}
$$

Since $|a|<1$ and (15) holds, the last equality shows that $\left|z_{0}\right|<1$ if and only if

$$
\left[9 \sin ^{2}\left(\alpha+\frac{\theta}{2}\right)+\cos ^{2}\left(\alpha+\frac{\theta}{2}\right)\right]|a|^{2}<1,
$$


and $\left|z_{0}\right|=1$ if and only if

$$
\left[9 \sin ^{2}\left(\alpha+\frac{\theta}{2}\right)+\cos ^{2}\left(\alpha+\frac{\theta}{2}\right)\right]|a|^{2}=1 .
$$

In conclusion, if (14) and (15) hold, then $-A \in \mathbb{D}$ and $-B \in \overline{\mathbb{D}}$. Thus, by virtue of Lemma 2 , we obtain that the dilatation $\widetilde{\omega}$ of $f_{0} * f$ satisfies

$$
|\widetilde{\omega}(z)|=\left|\frac{z(z+A)(z+B)}{(1+\bar{A} z)(1+\bar{B} z)}\right|<1 \quad(z \in \mathbb{D}) .
$$

Furthermore, by Theorem A, we conclude that $f_{0} * f \in \mathcal{S}_{H}^{0}$ and is convex in the direction of the real axis.

Remark 1. If we give different valves for the parameters $\theta \in \mathbb{R}$ and $a \in \mathbb{D}$ in Theorem 1, then we obtain the results Theorem B, Theorem C, and the sufficiency part of Theorem D, respectively.

Finally, we present an example of a function, and the corresponding convolution function with a given dilatation, and illustrate them graphically by using Mathematica software package. The example illustrates how Theorem 1 generalizes the result of Theorem D.
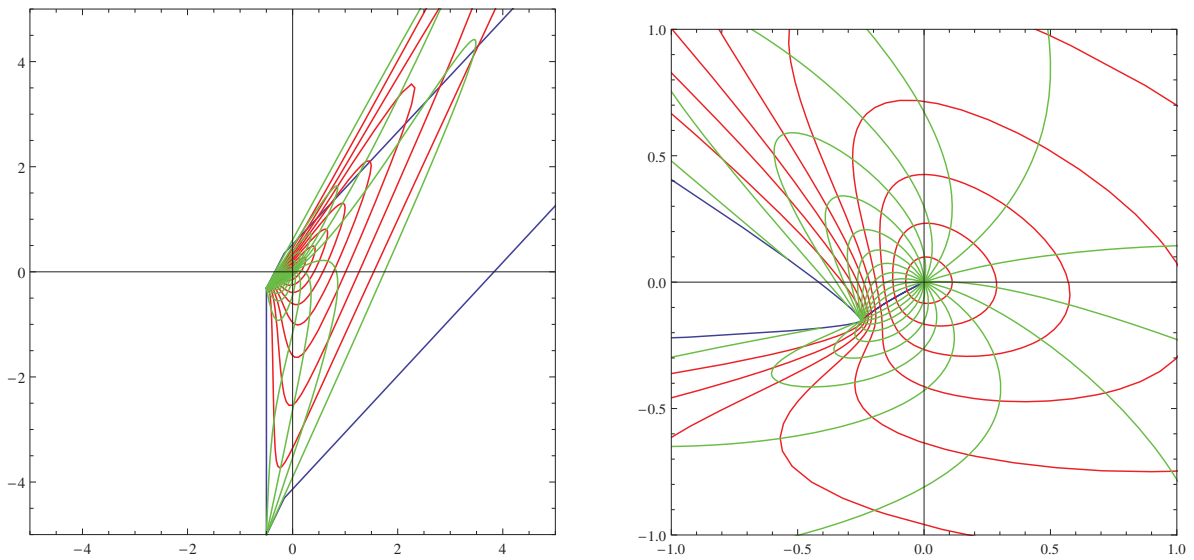

Figure 1. The images of $\mathbb{D}$ under the mappings $f$ and $f_{0} * f$ (right).

Example 1. If $f=h+\bar{g} \in \mathcal{R}_{H}^{0}$, and the dilatation is given by

$$
\omega(z)=\frac{z+a}{1+\bar{a} z}
$$

with $a=i / 2$, then obviously, the conditions (5) are not satisfied and Theorem $\mathrm{D}$ cannot be used. 
However, if $f=h+\bar{g} \in \mathcal{R}_{H}^{0}$, and the dilatation $\omega(z)$ is given by (6) with $a=i / 2$ and $\theta=-\pi$, then $\alpha=\pi / 2$, and the conditions

$$
\left[9 \sin ^{2}\left(\alpha+\frac{\theta}{2}\right)+\cos ^{2}\left(\alpha+\frac{\theta}{2}\right)\right]|a|^{2}=|a|^{2}=\frac{1}{4}<1
$$

and

$$
|a| \cos \left(\alpha+\frac{\theta}{2}\right)=|a|=\frac{1}{2} \neq 0=-\cos \frac{\theta}{2}
$$

are satisfied. Thus, by Theorem $1, f_{0} * f \in \mathcal{S}_{H}^{0}$ and is convex in the direction of the real axis. Next we illustrate this result by finding the images of $\mathbb{D}$ under the mappings $f$ and $f_{0} * f$.

First, we need to get the expressions of $h$ and $g$. Since

$$
h(z)+g(z)=\frac{z}{1-z} \quad \text { and } \quad g^{\prime}(z)=\frac{2 z+i}{i z-2} h^{\prime}(z)
$$

we obtain

$h^{\prime}(z)=\frac{i z-2}{(1-z)^{2}[(i+2) z+(i-2)]} \quad$ and $\quad g^{\prime}(z)=\frac{2 z+i}{(1-z)^{2}[(i+2) z+(i-2)]}$.

Computing $h(z)$ and $g(z)$ yields

$$
\begin{aligned}
& h(z)=\frac{1+2 i}{2} \frac{z}{1-z}+\frac{3}{4} \log \frac{(i+2) z+(i-2)}{(i-2)(1-z)}, \\
& g(z)=\frac{1-2 i}{2} \frac{z}{1-z}-\frac{3}{4} \log \frac{(i+2) z+(i-2)}{(i-2)(1-z)} .
\end{aligned}
$$

By writing the convolution, we have

$$
f_{0} * f=h_{0} * h+\overline{g_{0} * g}=\frac{h+z h^{\prime}}{2}+\frac{\overline{g-z g^{\prime}}}{2},
$$

so that

$$
h_{0} * h=\frac{1+2 i}{4} \frac{z}{1-z}+\frac{3}{8} \log \frac{(i+2) z+(i-2)}{(i-2)(1-z)}+\frac{(i z-2) z}{2(1-z)^{2}[(i+2) z+(i-2)]}
$$

and

$g_{0} * g=\frac{1-2 i}{4} \frac{z}{1-z}-\frac{3}{8} \log \frac{(i+2) z+(i-2)}{(i-2)(1-z)}-\frac{(2 z+i) z}{2(1-z)^{2}[(i+2) z+(i-2)]}$.

Applying Lemma 2 with $\omega(z)=\frac{2 z+i}{i z-2}$, we get

$$
|\widetilde{\omega}(z)|=\left|\frac{z t(z)}{t^{*}(z)}\right|=\left|\frac{z(z+A)(z+B)}{(1+\bar{A} z)(1+\bar{B} z)}\right|<1,
$$

where

$$
-A \approx 0.776257-0.592964 i \text { and }-B \approx-0.476257-0.557036 i
$$

are the roots of the equation

$$
t(z)=z^{2}-\frac{1}{20}(6-23 i) z-\frac{1}{20}(14+3 i)=0 .
$$


The images of the unit disk $\mathbb{D}$ under $f$ and $f_{0} * f$ are shown in Figure 1 .

Acknowledgements. The authors would like to thank the referee for the helpful comments and suggestions. The research was supported by National Natural Science Foundation of China (Grant No. 11371126) and Academy of Finland and National Natural Science Foundation of China (Grant No. 269260).

\section{References}

[1] O. P. Ahuja and J. M. Jahangiri, Convolutions for special classes of harmonic univalent functions, Appl. Math. Lett. 16 (2003), no. 6, 905-909.

[2] R. M. Ali, B. A. Stephen, and K. G. Subramanian, Subclass of harmonic mappings defined by convolution, Appl. Math. Lett. 23 (2010), no. 10, 1243-1247.

[3] D. Bshouty and A. Lyzzaik, Problems and conjectures in planar harmonic mappings, J. Anal. 18 (2010), 69-81.

[4] J. Clunie and T. Sheil-Small, Harmonic univalent functions, Ann. Acad. Sci. Fenn. Ser. A. I Math. 9 (1984), 3-25.

[5] A. Cohn, Über die Anzahl der Wurzeln einer algebraischen Gleichung in einem Kreise, Math. Z. 14 (1922), no. 1, 110-148.

[6] M. Dorff, Convolutions of planar harmonic convex mappings, Complex Var. Theory Appl. 45 (2001), no. 3, 263-271.

[7] M. Dorff, M. Nowak, and M. Wołoszkiewicz, Convolutions of harmonic convex mappings, Complex Var. Elliptic Equ. 57 (2012), no. 5, 489-503.

[8] P. Duren, Harmonic Mappings in the Plane, Cambridge University Press, Cambridge, 2004.

[9] M. Goodloe, Hadamard products of convex harmonic mappings, Complex Var. Theory Appl. 47 (2002), no. 2, 81-92.

[10] R. Kumar, S. Gupta, S. Singh, and M. Dorff, On harmonic convolutions involving a vertical strip mapping, Bull. Korean Math. Soc. 52 (2015), no. 1, 105-123.

[11] L.-L. Li and S. Ponnusamy, Solution to an open problem on convolutions of harmonic mappings, Complex Var. Elliptic Equ. 58 (2013), no. 12, 1647-1653.

[12] Convolutions of slanted half-plane harmonic mappings, Analysis (Munich) 33 (2013), no. 2, 159-176.

YUE-PING JIANG

School of Mathematics and ECONOMEtrics

Hunan University

Changsha 410082, Hunan, P. R. China

E-mail address: ypjiang731@163.com

Antti Rasila

Department of Mathematics and Systems Analysis

Aalto University

P. O. Box 11100, FI-00076 Aalto, Finland

E-mail address: antti.rasila@iki.fi

YONG SUN

School of Mathematics and EConometrics

HUNAN UNIVERSITY

Changsha 410082, Hunan, P. R. China

E-mail address: yongsun2008@foxmail.com 